\title{
Gene-expression profiling of microdissected breast cancer microvasculature identifies distinct tumor vascular subtypes
}

François Pepin ${ }^{1,2,3,4,5}$, Nicholas Bertos ${ }^{3,4 \dagger}$, Julie Laferrière ${ }^{3,4 \dagger}$, Svetlana Sadekova ${ }^{3,6}$, Margarita Souleimanova ${ }^{3,4}$, Hong Zhao ${ }^{3,4}$, Greg Finak ${ }^{1,2,3}$, Sarkis Meterissian ${ }^{8}$, Michael T Hallett ${ }^{1,2,4}$ and Morag Park ${ }^{1,3,4,9^{*}}$

\begin{abstract}
Introduction: Angiogenesis represents a potential therapeutic target in breast cancer. However, responses to targeted antiangiogenic therapies have been reported to vary among patients. This suggests that the tumor vasculature may be heterogeneous and that an appropriate choice of treatment would require an understanding of these differences.
\end{abstract}

Methods: To investigate whether and how the breast tumor vasculature varies between individuals, we isolated tumor-associated and matched normal vasculature from 17 breast carcinomas by laser-capture microdissection, and generated gene-expression profiles. Because microvessel density has previously been associated with disease course, tumors with low $(n=9)$ or high $(n=8)$ microvessel density were selected for analysis to maximize heterogeneity for this feature.

Results: We identified differences between tumor and normal vasculature, and we describe two subtypes present within tumor vasculature. These subtypes exhibit distinct gene-expression signatures that reflect features including hallmarks of vessel maturity. Potential therapeutic targets (MET, ITGAV, and PDGFR $\beta$ ) are differentially expressed between subtypes. Taking these subtypes into account has allowed us to derive a vascular signature associated with disease outcome.

Conclusions: Our results further support a role for tumor microvasculature in determining disease progression. Overall, this study provides a deeper molecular understanding of the heterogeneity existing within the breast tumor vasculature and opens new avenues toward the improved design and targeting of antiangiogenic therapies.

\section{Introduction}

The growth of tumors beyond a certain size requires the recruitment of an adequate blood supply, which is supplied by abnormal angiogenesis. This involves the triggering of an "angiogenic switch" [1], whereby the tumor microenvironment enters a proangiogenic mode in response to hypoxia. This process is accompanied by increased levels of multiple proangiogenic factors, including vascular endothelial growth factor A (VEGFA) and platelet-derived growth factor B (PDGFB), as well as decreases in antiangiogenic factors such as endostatin.

\footnotetext{
* Correspondence: morag.park@mcgill.ca

+ Contributed equally

'Department of Biochemistry, McGill University, 3655 Promenade Sir William Osler, Montréal, Québec, H3G 1Y6, Canada

Full list of author information is available at the end of the article
}

This leads to an increase in the number of proliferating endothelial cells, along with enhanced endothelial cell recruitment and migration toward the tumor bed [2].

Because endothelial cells are considered to be genetically stable [2], modulation of angiogenic processes is considered to be a promising area for cancer therapy. However, clinical trials of antiangiogenic therapies in breast cancer have reported mixed results [3-5]. Heterogeneity among tumor vasculatures may partially explain this lack of consistency [3]. The most common measure of vascular heterogeneity is microvessel density (MVD), which has weak prognostic value in breast cancer [6]. MVD is significantly associated with histologic type, but does not appear to be linked to other features, including nodal status, tumor grade, estrogen receptor (ER) status, molecular subtype, or

\section{Biomed Central}

(c) 2012 Pepin et al.; licensee BioMed Central Ltd. This is an open access article distributed under the terms of the Creative Commons Attribution License (http://creativecommons.org/licenses/by/2.0), which permits unrestricted use, distribution, and reproduction in any medium, provided the original work is properly cited. 
the presence of vascular invasion $[7,8]$. However, other characteristics of the tumor vasculature, such as vascular proliferation and levels of circulating endothelial progenitor cells, also have clinical significance in cancer $[9,10]$. Therefore, a more complete understanding of vascular heterogeneity is required.

Gene-expression profiling studies have provided a greater understanding of breast tumor heterogeneity $[11,12]$ and have highlighted the role of the stroma in influencing disease outcome [13-16]. However, previous studies of tumor vasculature have mostly been limited to small numbers of samples and have focused on direct comparisons between tumor-associated and normal vasculature [17-23]. Although these studies have identified alterations characteristic of the tumor vasculature perse, they have not addressed the question of vascular heterogeneity or investigated whether distinct intertumoral vascular subtypes exist. Here, we use laser-capture microdissection (LCM) to isolate tumor-associated and matched normal microvascular compartments separately from 17 human breast cancer samples. After gene-expression profiling, we identified and validated gene-expression signatures that define two distinct subtypes of tumor vasculature, an understanding of which would represent an important step toward the improved design and targeting of therapeutic modalities.

\section{Materials and methods}

Tissue samples from 21 patients undergoing surgery for primary invasive ductal carcinoma (IDC) with no prior neoadjuvant therapy, and from one patient undergoing reduction mammoplasty, were subjected to LCM (see Additional File 1, Table S1A, B). From this cohort, we obtained 17 samples of tumor-associated vasculature; in 15 cases, we also obtained normal vasculature. After determination of MVD (see Additional File 2, Figure S1A), PECAM1-positive cells (microvasculature) were collected by using LCM from within tumor beds $(n=17)$, as well as from morphologically normal tissue adjacent to breast tumors $(n=14)$ and from one reduction mammoplasty (sample V) (see Additional File 2, Figure S1B). Only PECAM1-positive cells present within capillaries and small vascular structures were collected, avoiding isolated PECAM1-positive cells and vessels with large lumens. Total RNA was extracted, amplified, labeled, and hybridized on Agilent 44K Whole Genome arrays (Agilent Technologies, Santa Clara, CA). The endothelial and epithelial cell content of isolated samples was assessed by quantitative real-time PCR (qRT-PCR) (see Additional File 2, Figure S1C).

\section{Description of samples}

Clinical information was collected on a prospective basis (median follow-up, 5.6 years; see Additional File 1,
Table S1B). Occurrence of distant recurrence was determined by examination of medical records. Matched normal epithelial samples were obtained as previously described [24]. This study was approved by the McGill University Health Centre Research Ethics Board (Protocols SDR-99-780 and SDR-00-966). All patients provided written, informed consent.

\section{Laser-capture microdissection, RNA isolation, and microarray hybridization}

Immunohistochemistry directed against PECAM1 for MVD quantitation was carried out as per the antibody manufacturer's instructions. Quantitation of PECAM1 staining density was performed by averaging the stained pixel intensity of three fields captured at $10 \times$ magnification by using imageJ [25]. Details of the anti-PECAM1 immunohistochemistry protocol used for guided LCM are presented in Supplementary Information (see Additional File 3, Supplementary Materials and Methods). LCM, RNA isolation and sample preparation, as well as microarray hybridization, were carried out as previously described [13]. Microarray results have been submitted to the Gene Expression Omnibus (GSE15363).

\section{Differential expression, class discovery, and class prediction}

All analyses were performed using R/Bioconductor version 2.6 [26]. Microarray data were extracted as described in Supplementary Information (see Additional File 3, Supplementary Materials and Methods). Initially, the set of most-variable probes $(n=1,168)$ was defined as those with an interquartile range above 2 . Class discovery was performed by hierarchical clustering based on this set, using Ward's minimum-variance method with a correlation distance metric. The significance of the resulting clusters was calculated by using the pvclust package with 10,000 bootstrap iterations. Heatmaps were generated by scaling each row (gene) into Z-scores, subtracting the mean and dividing by its standard deviation. Differential expression between sample sets was determined by using Linear Models for Microarray Analysis (LIMMA) [27]. For genes represented by multiple probes, only the probe with the largest interquartile range was used. Unless stated otherwise, a gene was considered differentially expressed if the FDR-adjusted $P$ value was $\leq 0.05$ [28].

Predictors of recurrence were generated as follows: gene expression for subtype B member profiles was adjusted by removing the average differences between subtype B and subtype A tumor vascular expression for each gene. Then, the six most significantly differentially expressed genes between recurrent and nonrecurrent tumor vasculature were identified by using a Wilcoxon rank-sum test. These genes were used to train a linear discriminant analysis classifier and tested for accuracy 
through leave-one-out cross-validation (LOOCV). All steps (including the adjustment of tumor vascular subtypes) were done under cross-validation. Gene sets of size two to 100 were tested for prediction, and six-gene predictors were found to exhibit the highest accuracy under LOOCV.

\section{Quantitative real-time PCR and immunohistochemistry}

Validation for selected genes and cognate proteins was performed by qRT-PCR and IHC, respectively, on subsets of samples. Full details and probe sequences are presented in Supplementary Information (see Additional File 3, Supplementary Materials and Methods).

\section{Results and discussion}

Because our goal was to investigate the heterogeneity of the tumor microvasculature in invasive ductal carcinomas, samples with high and low MVD were chosen to maximize the spectrum of heterogeneity present within the sample cohort. Within this set, we identified tumor samples with high $(n=8)$ or low $(n=9)$ MVD, as determined by immunohistochemistry directed against the endothelial marker PECAM1 (see Additional File 1; Table S1B; Additional File 2, Figure S1A).

\section{Isolation of vasculature-enriched samples and gene- expression profiling}

A significant enrichment of PECAM1 was observed in microdissected vasculature when compared with the epithelial cell marker cytokeratin 8 (KRT8); conversely, the latter marker was enriched in matched microdissected epithelial RNA samples (see Additional File 2, Figure S1C). These results indicate that the vascular samples are highly enriched in endothelial cell content.

\section{Tumor and normal vasculatures possess distinct expression profiles}

To investigate the differences among the expression profiles generated, we performed hierarchical clustering by using the 1,168 most variable genes (interquartile range $>2$ ) over the entire dataset. The normal and tumor vascular samples formed two distinct clusters (Figure 1A; see

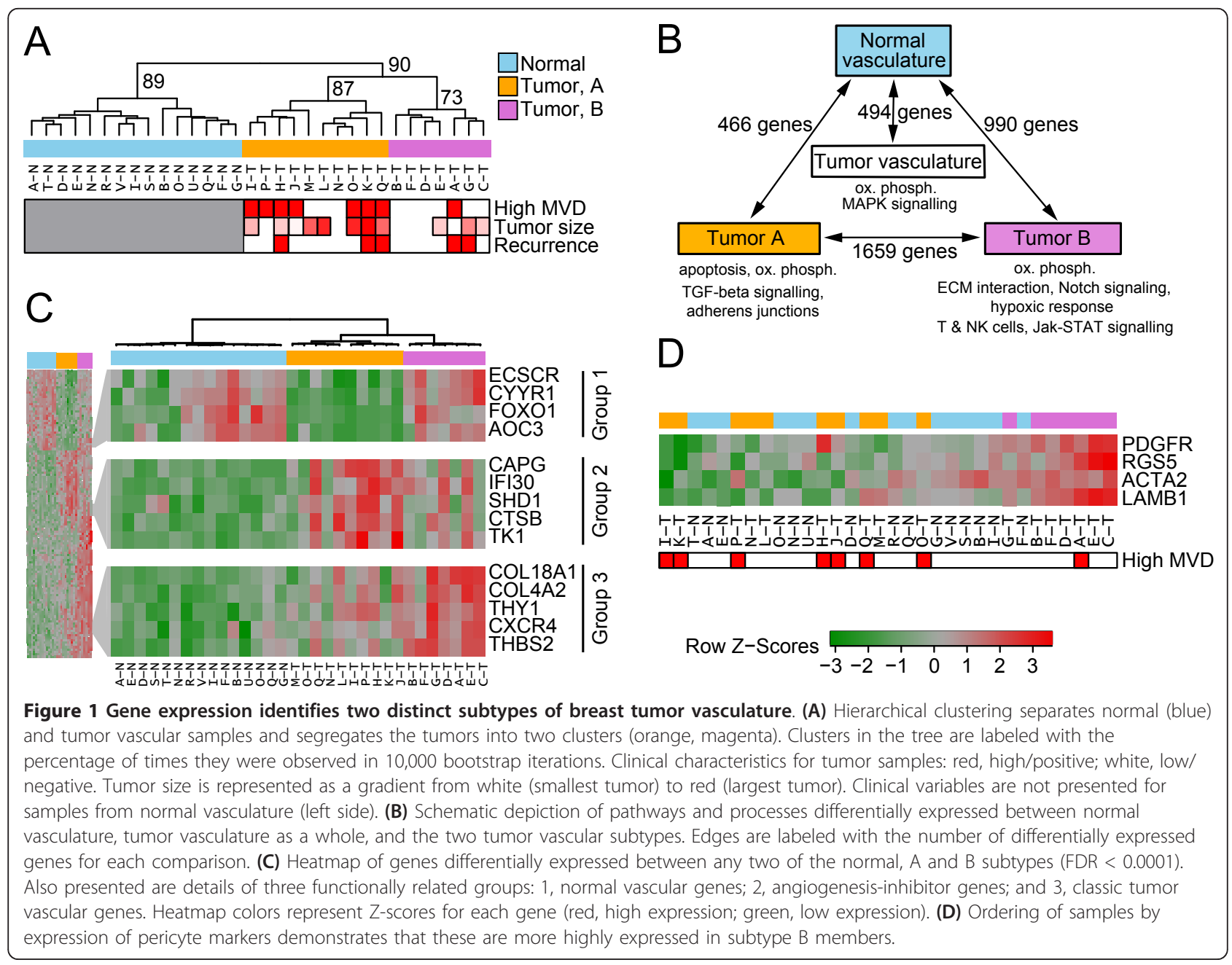


Additional File 2, Figure S2A). Further comparison of the tumor and normal vasculature samples identified 494 differentially expressed genes (FDR $<0.05$, LIMMA; see Additional File 4, Sheet 1).

A significant overlap exists between the gene sets discriminating between tumor and normal vasculature identified by previous studies [17-23] and here $(P<0.05$ for each, Fisher Exact test; Figure S3A). This overlap is significant even for the studies performed on different tumor types [19-23] or by using other isolation techniques [18-20,23]. Common processes include extracellular matrix remodeling and cytokine signaling [29] (see Additional File 1, Table S2). Several genes previously reported to be elevated in tumor vasculature [29] are also elevated in our tumor vascular cohort, including osteonectin (SPARC), collagens 1A1, 3A1, 4A2, and 18A1 (COL1A1, COL3A1, COL4A2 and COL18A1), and Thy cell-surface antigen (THY1) (see Additional File 4, Sheet 1). This is consistent with the observation that tumor vasculature is constantly reorganizing $[30,31]$ and confirms that our normal and tumor vasculature are comparable to those isolated in previous studies.

The tumor vasculature also exhibits differential expression of genes related to oxidative phosphorylation/stress and MAPK signaling (Figure 1B; see Additional File 1, Table S2; Additional File 4, Sheet 1). It is known that reoxygenation of vasculature, consistent with the variable flow of blood in tumor vessels, leads to the creation of reactive oxygen species [32].

\section{Tumor vascular expression profiles segregate into two} distinct subtypes reflecting different biological processes Interestingly, the hierarchical clustering revealed that the tumor samples form two distinct clusters, here termed A and B (Figure 1A; see Additional File 1, Figure S2A); this separation was also reflected in the first component when the set of tumor vascular samples were subjected to principal-component analysis (see Additional File 1, Figure S2B). Analysis of differential expression between these two clusters identifies 1,659 genes (FDR $<0.05$, LIMMA; see Additional File 4, Sheet 2). This is more than 3 times larger than the number of genes differentially expressed between normal and tumor samples, emphasizing the significance of the clusters and indicating that homogeneity within each cluster is higher than that within the tumor vasculature as a whole. Thus, separating tumor samples by cluster may prove more informative than grouping them together.

The tumor vascular clusters are independent of major clinical variables, including ER and HER2 status, grade, and lymph node involvement $(P \geq 0.6$; see Additional File 1 , Table S1A) but are enriched for larger and smaller tumors as well as high and low MVD $(P<0.05, t$ test). Interestingly, no statistically significant differential gene expression was found between high- and low-MVD samples (FDR $<0.05$, LIMMA). This demonstrates that although MVD status is associated with the tumor vascular clusters identified here, additional properties of the samples are primarily responsible for the molecular profiles and observed clustering.

To identify biological functions specifically associated with each of the two subtypes of tumor vasculature, we identified the most highly differentially expressed genes (FDR < 0.0001, LIMMA, Figure 1C; FDR < 0.05, LIMMA; see Additional File 4, Sheet 2) and pathways (FDR $<0.05$; Figure 1B; see Additional File 1, Table S2) between normal vasculature and each of the tumor vascular subtypes $\mathrm{A}$ and $\mathrm{B}$.

Genes within Group 1 exhibit the highest expression in normal samples and the lowest expression in tumorassociated vasculature subtype A members (Figure $1 \mathrm{C}$ ). Interestingly, these include $A O C 3$ and FOXO1, both associated with vascular shear stress [33,34]. Reduced shear stress suggests a decreased flow rate, consistent with the hypothesis that tumor vessels, and in particular those belonging to subtype A, lack appropriate perfusion [35]. Many genes and processes related to metabolism and biosynthesis are also overrepresented in subtype A (see Additional File 1, Table S2; Additional File 4, Sheet 2 ), suggesting active proliferation. Consistent with this, Group 2, consisting of genes whose expression is elevated in subtype A (Figure $1 C$ ), includes cathepsin B $(C T S B)$, associated with active vascular remodeling $[35,36]$.

Genes within Group 3 are more highly expressed in subtype B, and are enriched for elements involved in interactions with the extracellular matrix, collagen production, focal adhesion, hypoxia, glycolysis, immune response, and protein export, suggesting that this group may represent a more stable vasculature (Figure $1 \mathrm{C}$; Additional File 1, Table S2). These include genes involved in antiangiogenic processes, such as thrombospondin 2 (THBS2) and the collagens COL18A1 and COL4A2, which can be cleaved to form endostatin and canstatin, respectively [37]. Antiangiogenic signaling from these and other genes (see Additional File 1, Figure S3B) may contribute to the decreased overall MVD seen in these samples. Interestingly, NOTCH3 is also elevated in subtype B (see Additional File 4, Sheet 2); Notch signaling is implicated in vascular development and maturation [38,39].

Tumor vasculature is often considered immature with respect to normal vasculature [30], the known functions of many of the individual genes identified here suggest that the two tumor vascular clusters may represent differences in degree of vascular maturity. This hypothesis was investigated by using several approaches. Pericytes line mature capillary vessels and would be co-isolated with PECAM1-positive endothelial cells during LCM, 
whereas the association of pericytes with immature vessels is relatively loose, and this cell type would therefore be co-isolated at a lower frequency in such vessels [31]. Therefore, we explored whether clusters A and B differed with respect to their content of pericyte-specific signals, specifically the pericyte markers ACTA2, $P D G F R \beta$, and RGS5 [40], as well as $L A M B 1$, a surrogate of laminin-8 $(\alpha 4 \beta 1 \gamma 1)$ implicated in vessel maturation [41] (Figure 1D). Interestingly, these markers are elevated in cluster $\mathrm{B}(P<0.05$ for each; $t$ test), and their expression pattern successfully recapitulates the clusters (Figure 1D). Differential expression of PDGFR $\beta$ was confirmed by qRT-PCR on amplified RNA prepared from tumor vascular samples (see Additional File 2, Figure S3C), whereas immunohistochemistry (IHC)-based validation was also performed for ACTA2 and LAMB1 (Figure 2A, B; see Additional File 2, Figure S4A). Serial staining directed against PECAM1 showed that most ACTA2 and LAMB1 expression is observed in proximity to the PECAM1-expressing vasculature (see Additional File 2, Figure S4B, C), while pixel counts over whole sections (normalized for PECAM1 staining, as assessed on a neighboring section) showed a significantly increased expression of ACTA2 and LAMB1 in tumors belonging to cluster B $(P<0.05$ for each, Wilcoxon test; Figure 2C; Additional File 1, Table S1A). Therefore, the differential gene expression observed between clusters is recapitulated at the protein level for these markers. In addition, assessment of $L A M B 1$ mRNA content in selected samples by qRT-PCR significantly correlated with the microarray-derived values; this also held true for several other selected genes (see Additional File 2, Figure S5). Supporting this observation, a negative correlation between MVD and pericyte coverage has also been identified in endometrial cancer [9].

To assess whether the subtype B-specific gene-expression profile is driven by pericyte-specific genes, the sample orderings induced by pericyte marker expression and

\section{A

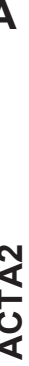

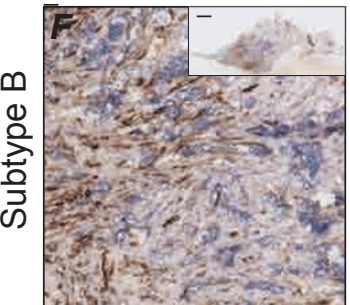
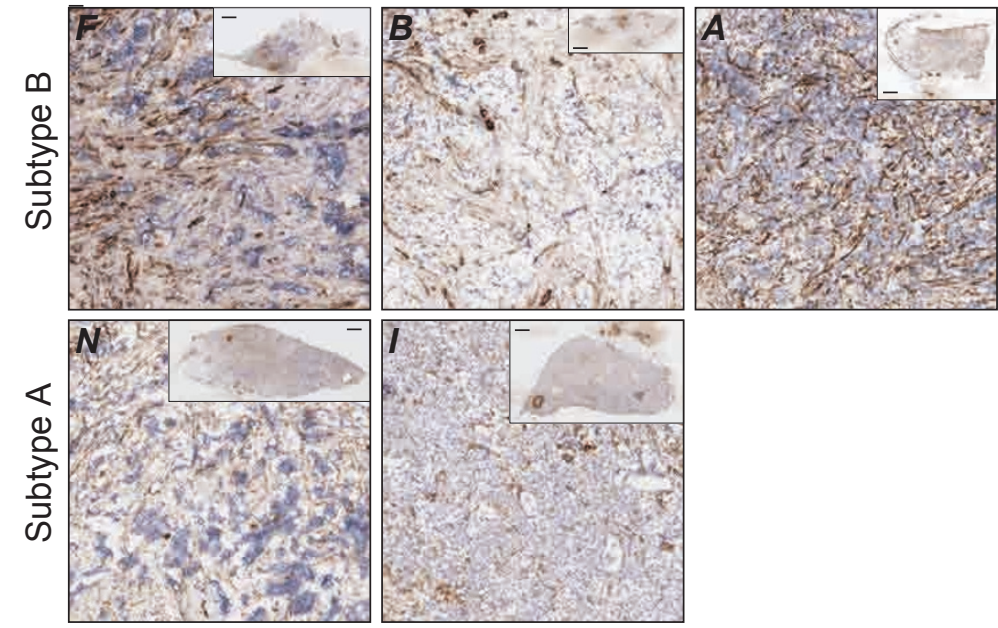

B

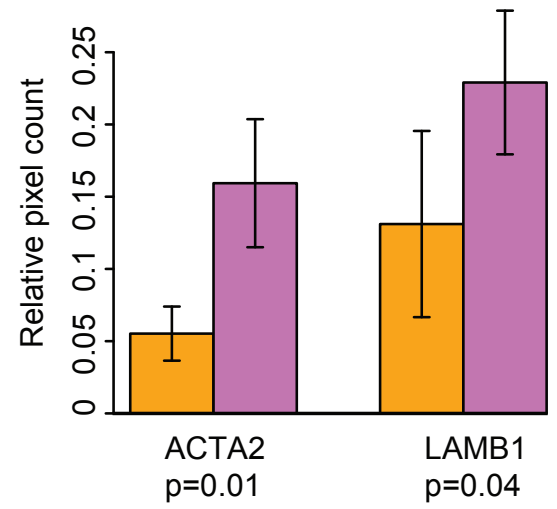

Tumor, A

Tumor, B

Figure 2 Immunohistochemistry directed against selected subtype markers confirms their differential expression between subtypes. (A) Anti-ACTA2 immunohistochemistry. Individual sample labels are indicated in italic font in the upper left corner of each image. Each image is of a representative $1 \times 1-\mathrm{mm}$ area; inset depicts whole section; scale bar in inset, $1 \mathrm{~mm}$. (B) Bar plot of pixel counts for IHC staining of ACTA2 and $L A M B 1$, relative to PECAM1 staining. 
subtype-specific gene expression were compared (see Additional File 2, Figure S6). First, we confirmed that ordering of all samples by relative expression of known pericyte markers recapitulated the separation of subtype A and B members (Additional File 2, Figure S6A, B); this ordering was not correlated with expression of endothelial marker genes (Additional File 2, Figure S6C). Subtype B members were then ordered by relative expression of known pericyte markers (Additional File 2, Figure S6D). Then, retaining this ordering, the relative expression of the top 200 genes differentially expressed between the A and B subtypes (ranked by $P$ value) was examined (see Additional File 2, Figure S6E). Importantly, no correlation was seen at the sample level between relative pericyte-marker content and relative expression levels of subtype-specific genes within the set of subtype B members, supporting that processes other than those linked to pericyte-marker content distinguish these two subtypes.

Pathway analysis highlights an increased expression of genes related to glycolysis and oxidative phosphorylation in subtype B (Figure 1B). This is intriguing, as elevation of either glycolysis or oxidative phosphorylation often leads to downregulation of the other [42]. Coupled with an increased expression of ribosomal genes, the increase in oxidative phosphorylation could be evidence of increased metabolism in subtype B vasculature. Interestingly, the different tumor vascular subtypes also vary at the level of NK and T-cell marker expression, suggesting that vascular subtypes can affect the tumor microenvironment. Additionally, CXCL12/SDF-1, considered to play a role in pericyte recruitment [43], is decreased in subtype A tumor vasculature relative to subtype B, consistent with the presence of increased levels of pericyte markers in subtype $\mathrm{B}$, whereas angiopoietin-2 (ANGPT2), thought to promote blood-vessel destabilization by acting as an ANGPT1 antagonist [44], is elevated in subtype B tumor vasculature.

Together, these results strongly support the hypothesis that the vascular clusters observed here reflect differences in vessel maturity, and indicate that related processes, including anti- and proangiogenic regulators and matrix remodeling, also contribute to the segregation of the vascular subtypes. We suggest that cluster B, associated with low MVD, may represent samples with a more mature tumor vasculature with respect to members of cluster A. Other potential factors driving cluster segregation may include unidirectional shear stress (USS), expected to be highest in vessels of subtype B. USS elicits an antiinflammatory response [45] and has been shown to induce miR-21 expression in endothelial cells, leading to decreased apoptosis and activation of the nitric oxide pathway, implicated in Notch signaling [46]. All of these are consistent with our proposed subtypes (Figure 1B). Interestingly, genes previously identified as being commonly overexpressed in tumor endothelium and therefore proposed as specific markers for endothelial cell targeting, including SPARC and COL4A1 [47], are elevated in subtype B but not subtype A tumor vasculature, accentuating the importance of gaining a better understanding of the heterogeneity present in this compartment.

\section{Most previous signatures of tumor vascular cells identify only tumor vascular subtype B}

Previous studies (see Additional File 2, Figure S3A) have generated signatures that segregate tumor versus normal vascular samples, but have not identified distinct subtypes within the tumor vasculature. To test whether these signatures can differentiate between our tumor vascular subtypes, we examined the clustering induced by previously reported tumor vascular signatures $[17,18,21-23]$ in our dataset. All previously published tumor vascular gene sets investigated could segregate members of subtype B from normal samples in our data (Figure 3; Additional File 2, Figure S3D-G); however, only the gene set described by Bhati et al. [17] generated from breast tumors using LCM, successfully segregates the subtype A and normal samples (Figure 3A). The inability of most existing datasets to generate signatures capable of distinguishing between tumor vascular subtypes may be partially explained by differences in the isolation technique used. Unlike LCM, immunomagnetic bead-based approaches using dispersed cells fail to isolate cells adjacent to the vessels that can provide additional information about the vascular microenvironment. Additionally, differences in the tissue of origin may contribute to these observations (see Additional File 2, Figure S3A), suggesting that subtype A may be enriched in breast tumors, and supporting the concept of tissuespecific antiangiogenic approaches.

The ability of the tumor vascular signature from Bhati et al. [17] to partially recapitulate our tumor vascular subtypes (Figure 3A) suggests that their samples contained features of both subtypes, and indicates that this vascular heterogeneity is likely to be common in breast cancer. Bhati et al. [17] used laser-capture microdissection to isolate factor VIII-expressing vascular cells from human breast cancers and normal breast tissue; however, they failed to identify subtypes within the tumor sample set. This may partly be due to the restricted sample size used in their study $(n=5)$, whereas the increased size of our patient cohort $(n=17)$, in combination with our selection based on MVD, likely enabled us to capture a broader range of interpatient variation in tumor vasculature. The small number of samples investigated in most previous studies ( $n=1$ to 5 ) $[17-20,22,23]$ also raises the possibility that only members of one subtype were present in their study cohorts. Hence our identification and initial characterization of distinct breast cancer vasculature subtypes is a novel finding that emphasizes the concept that breast 

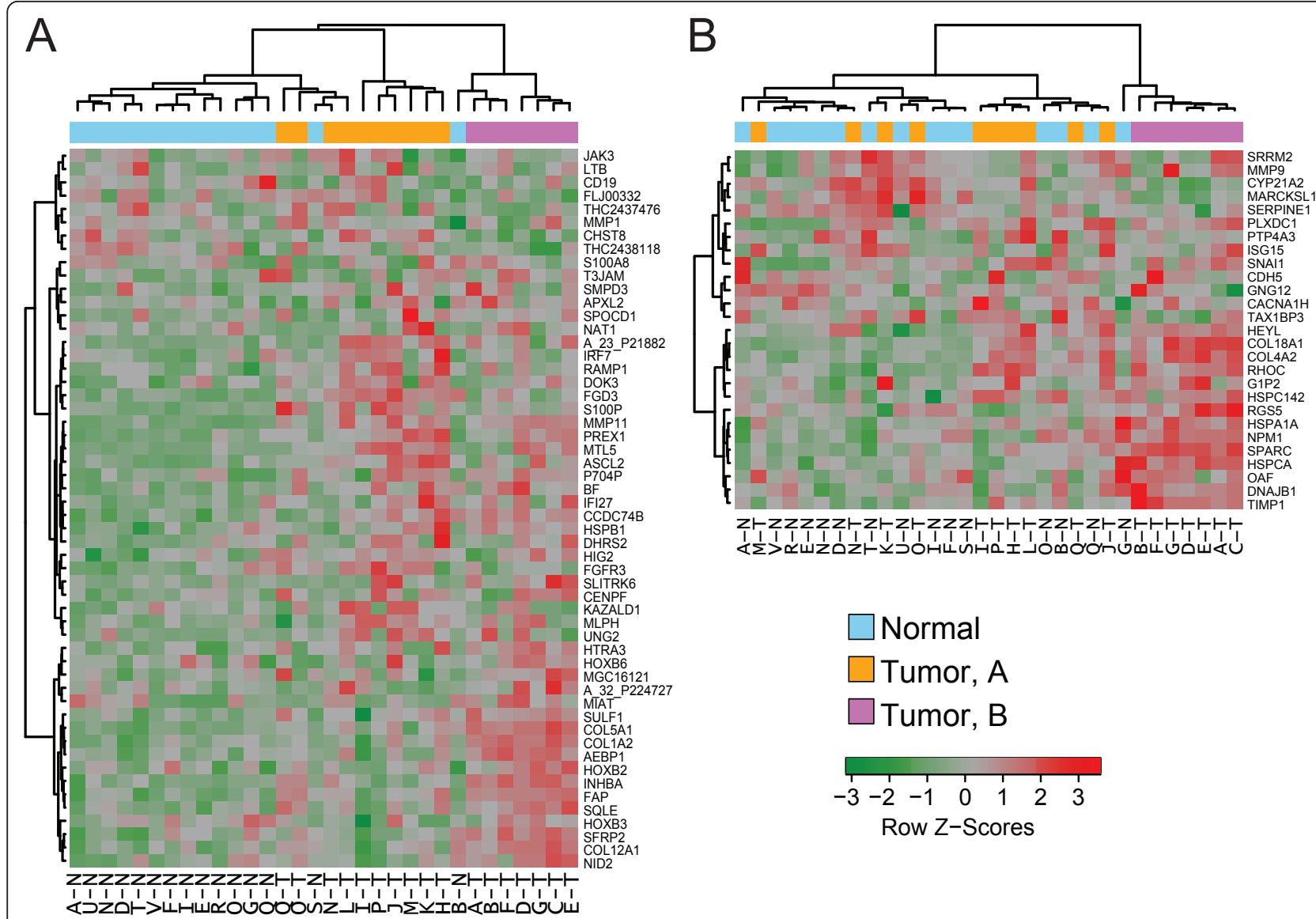

Figure 3 Tumor context and tissue of origin affects vascular expression in tumor samples. (A) The tumor vascular signature from Bhati et al. [17] separates tumor from normal vasculature in our samples, and also segregates most members of the A and B subtypes. (B) The tumor vascular signature from Parker et al. [18] identifies subtype B tumor vasculature, but fails to segregate subtype A tumor vasculature from normal samples.

cancer heterogeneity extends to the different subcomponents of the tumor microenvironment.

\section{Gene-expression differences induced by subtype membership mask differential gene expression associated with disease outcome}

To determine whether differences in tumor vasculature gene expression were correlated with other aspects of tumor biology and disease course, we performed differential expression analysis between the five recurrent and 12 nonrecurrent tumor samples in our dataset. This identified a total of 128 genes $(P<0.01$, no adjustment: see Additional File 1, Table S3), including TLR2 (involved in innate immunity) [48] and GAB1 (a scaffold protein linked to VEGF signaling and endothelial migration) [49]. Subtype membership is not correlated with recurrence in our relatively small dataset. This agrees with previous reports that differences in vessel maturity are associated with lymph node status but not other clinical variables in breast cancer [50]. However, we speculated that the differences in gene expression induced by subtype membership might be sufficiently large to mask differential gene expression associated with disease outcome. Similar conclusions have been drawn regarding gene expression-based predictors of breast cancer outcome based on whole-tumor data, which have generally been found to be more reliable when limited to specific tumor subtypes [51,52]. Generation of subtype-specific predictors was not possible, given the small size of each group and the limited number of recurrences in each (see Additional File 1, Table S1A,B). Therefore, we investigated this hypothesis by removing the gene-expression changes that could be ascribed to subtype membership. This was achieved by calculating the difference in average expression between the members of the two subtypes for each probe, and subtracting this value from the relevant probe data for all members of one subtype. After this adjustment, we could successfully build a six-gene predictor that identified recurrent patient samples with $94 \%$ accuracy under cross-validation in our dataset $(P=$ 0.002; Fisher Exact test; Figure 4A). Without applying this 


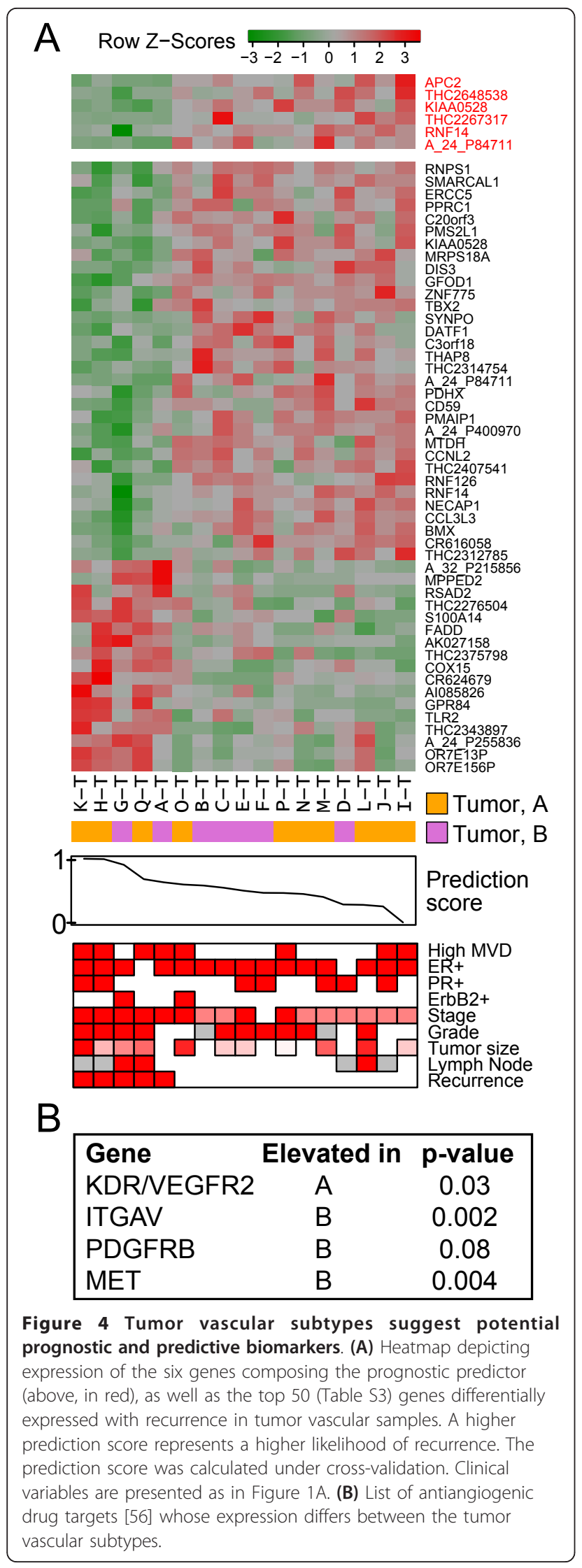

correction, no successful predictor could be constructed. The fact that the combination of these genes can predict recurrence under cross-validation within our dataset confirms their differential expression, despite their individual lack of significance after FDR adjustment.

This indicates that vascular markers of recurrence can be subtype-specific and that the subtypes contain different markers linked to recurrence. Additionally, the observation that successful construction of a predictor cannot be achieved without taking the inter-subtype differences into account suggests that these differences are sufficiently large to mask signals associated with other clinical variables, and supports that tumor vascular subtypes must be taken into account in future analyses of this compartment.

Our earlier work established that breast tumor stroma as a whole contains sufficient information to predict recurrence independently [13]. This study highlights that features of patient-specific vasculatures can reflect differences in the tumor microenvironment predictive of recurrence. As such, the tumor vasculature, including its subtypes, should be considered when studying the determinants of recurrence. This adds another dimension to previous reports that different characteristics of vasculature are associated with tumor stage and metastasis $[6,9,10,53]$, and indicates that the effects of tumor vasculature on disease progression are complex. Further research will be necessary to identify successfully all relevant factors contributed by the vascular environment in tumor progression.

\section{Drug targets are differentially expressed between subtypes}

The existence of vascular subtypes has important implications for the choice of antiangiogenic treatment strategies. A substantial proportion of patients in such clinical trials fail to show clinical benefits $[3,4]$; short-term treatment with one antiangiogenic drug led to an increased incidence of metastasis [54]. Importantly, observed differences between our subtypes (for example, pericyte coverage) may reflect mechanisms of resistance to antiangiogenic therapy [4]. Pericyte coverage can reduce tumor vessel vulnerability to VEGF inhibition, and in such cases, targeting treatments against both pericytes and endothelial cells may lead to improved efficacy [55].

The targets of several antiangiogenic drugs [56,57] are also differentially expressed between subtypes (Figure 4B). Both PDGFR $\beta$ and MET (a receptor tyrosine kinase, whose natural ligand HGF is a potent angiogenic factor [58]), are elevated in subtype $\mathrm{B}$; antagonists to $\alpha_{\mathrm{v}}$ integrin (ITGAV) [59], also elevated in subtype $B$, have been shown to block neovascularization. Vessel maturity has been linked to resistance to antiangiogenic therapy in melanoma [60], suggesting that this mechanism may be applicable across 
tumor types. The differential expression of drug targets indicates that subtype-specific approaches to targeting tumor angiogenesis may lead to improved response rates by permitting prestratification of patient populations. Modifications of the tumor vasculature, as suggested by a recent study identifying vessel normalization through ablation of the endothelial oxygen sensor PHD2 as a potential means to improve the delivery and efficacy of chemotherapeutic agents [61], may also have subtype-specific effects. The identification of biomarkers for prediction of response to antiangiogenic therapy is a current critical clinical need [62], and the vascular-subtype gene-expression profiles presented here provide new perspectives for the validation of such markers.

\section{Conclusions}

Overall, our data illustrate the heterogeneity of the breast tumor vasculature, define two tumor vascular subtypes that are associated with, but not defined by, microvessel density, and represent a first step toward establishing the landscape of vascular subtypes in breast cancer. Our previous work has shown that heterogeneity within the stromal compartment of breast tumors is linked to disease outcome [13]. We now suggest that breast tumor vasculature heterogeneity also plays a role in determining disease course, and that further study thereof may be critical for improved patient stratification before selection of specific targeted therapeutic regimens.

\section{Additional material}

\section{Additional file 1: Supplementary Tables S1 through S3} Supplementary Table S1A, B: Patient information. Supplementary Table S2. KEGG pathways differentially expressed between different categories of vasculature. Supplementary Table S3. List of genes differentially expressed between samples from recurrent and nonrecurrent cancer patients.

Additional file 2: Supplementary Figures S1 through S6. Supplementary Figure S1 Specific enrichment of the vasculature by laser capture microdissection. Supplementary Figure S2 Heatmap depicting differential gene expression after class discovery within the set of all microdissected endothelial samples and principal component analysis (PCA) of tumor endothelial samples. Supplementary Figure S3 Characteristics of the studies in which other tumor vascular signatures were generated, heatmaps representing expression of antiangiogenic genes and the pericyte marker PDGFR $\beta$ in tumor vasculature, and heatmaps of clustering induced by other tumor vascular signatures in the current dataset. Supplementary Figure $\mathbf{S} 4$ Images of anti-ACTA2 $I H C$, as well as of anti-ACTA2/LAMB1 and anti-PECAM1 IHC carried out on consecutive sections. Supplementary Figure S5 qRT-PCR validation for selected genes identified from analyses of microarray data. Supplementary Figure $\mathbf{S 6}$ Analyses of correlation between cell-type marker content and expression of genes differentially expressed between the $A$ and $B$ subtypes.

Additional file 3: Supplementary Materials and Methods. Supplementary Materials and Methods with corresponding references.

Additional file 4: Supplementary Datasets Sheets 1 and 2. Sheet 1: List of genes differentially expressed between normal and tumor vasculature. Sheet 2A-C: Lists of genes differentially expressed between normal vasculature and $A$ and $B$ tumor vascular-subtype members.

\section{Abbreviations}

ER: estrogen receptor; FDR: false discovery rate; HER2: human epidermal growth factor receptor 2; IHC: immunohistochemistry; KEGG: Kyoto Encyclopedia of Genes and Genomes; LCM: laser-capture microdissection; MVD: microvessel density; qRT-PCR: quantitative real-time polymerase chain reaction.

\section{Acknowledgements}

The authors thank D. Fleiszer, A. Loutfi, C. Milne, F. Tremblay, M. Wexler (surgeons), F. Halwani (pathologist), D. Hori, L. Pasyuk, T. Vilhena (pathology staff), and the MUHC Anaesthesia Department for their assistance with obtaining samples, as well as S. Dumont for technical support with LCM, S. Cory for bioinformatics support, and R. Annan for editing. The microarrays were hybridized and scanned by the Genentech Microarray Facility and the Genentech Bioinformatics department. H.P. Gerber coordinated the project at Genentech. This work was supported by grants from Meloche Monnex, the Québec Breast Cancer Foundation, and the Database and Tissue Bank Axis of the Réseau de recherche en cancer of the Fonds de la recherche en santé du Québec to MP; a Natural Science and Engineering Research Council of Canada Discovery Grants Program grant to MH; a Terry Fox New Frontiers Program grant to MP and MH; a U.S. Department of Defense Breast Cancer Predoctoral Traineeship Award to FP; Cedars Cancer Institute Fellowships to NB, JL, and SS; a L'Oréal Canada For Women in Science Research Excellence Fellowship, with the support of the Canadian Commission for UNESCO Program, to JL; and McGill University Health Centre Research Institute and Department of Medicine Fellowships to NB. We acknowledge infrastructure support and technical assistance from the Breast Cancer Functional Genomics Group, which is partially supported by funds from the Terry Fox New Frontiers Program. The funders had no role in study design, data collection and analysis, decision to publish, or preparation of the manuscript. MP holds the Diane and Sal Guerrera Chair in Cancer Genetics at McGill University.

\section{Author details}

'Department of Biochemistry, McGill University, 3655 Promenade Sir William Osler, Montréal, Québec, H3G 1Y6, Canada. ${ }^{2}$ McGill Centre for Bioinformatics, McGill University, 3649 Promenade Sir William Osler, Montréal, Québec, H3G OB1, Canada. ${ }^{3}$ Breast Cancer Functional Genomics Group, 1160 Ave. des Pins Ouest, Montréal, Québec, H3A 1A3, Canada. ${ }^{4}$ Rosalind and Morris Goodman Cancer Research Centre, McGill University, 1160 Ave. des Pins Ouest, Montréal, Québec, H3A 1A3, Canada. ${ }^{5}$ Sequenta, South San Francisco, CA 94080, USA. ${ }^{6}$ Merck, Palo Alto, CA 94304, USA. ${ }^{7}$ Fred Hutchinson Cancer Research Center, Seattle, WA 98101, USA. ${ }^{8}$ Department of Surgery, McGill University and Cedars Breast Clinic, McGill University Health Centre, 687 Ave. des Pins Ouest, Montréal, Québec, H3A 1A1, Canada. ${ }^{9}$ Department of Oncology, McGill University and Department of Medicine, McGill University Health Centre, 687 Ave. des Pins Ouest, Montréal, Québec, H3A 1A1, Canada.

\section{Authors' contributions}

FP participated in the design and coordination of the study, conducted bioinformatics analyses, and drafted the manuscript. NB supervised the later stages of the experimental work and helped to draft the manuscript. JL contributed to the later stages of the experimental work and helped to draft the manuscript. SS co-conceived of the study and supervised the initial phases of the experimental work. MS conducted immunohistochemistry and laser-capture microdissection experiments. $\mathrm{HZ}$ conducted RNA isolation, amplification, microarray, and GRT-PCR experiments. GF assisted with bioinformatics analyses and helped to draft the manuscript. SM provided clinical insights and assisted with obtaining samples. MTH co-supervised the design and coordination of the study, supervised bioinformatics analyses, and helped to draft the manuscript. MP conceived of the study, supervised its design and coordination, and helped to draft the manuscript. All authors read and approved the final manuscript.

\section{Competing interests}

The authors declare that they have no competing interests. 
Received: 27 April 2012 Revised: 16 July 2012

Accepted: 14 August 2012 Published: 20 August 2012

\section{References}

1. Hanahan D, Folkman J: Patterns and emerging mechanisms of the angiogenic switch during tumorigenesis. Cell 1996, 86:353-364.

2. Folkman J: Angiogenesis. Annu Rev Med 2006, 57:1-18.

3. Longo R, Gasparini G: Challenges for patient selection with VEGF inhibitors. Cancer Chemother Pharmacol 2007, 60:151-170.

4. Bergers $G$, Hanahan D: Modes of resistance to anti-angiogenic therapy. Nat Rev Cancer 2008, 8:592-603.

5. Carpini JD, Karam AK, Montgomery L: Vascular endothelial growth factor and its relationship to the prognosis and treatment of breast, ovarian, and cervical cancer. Angiogenesis 2010, 13:43-58.

6. Uzzan B, Nicolas P, Cucherat M, Perret G-Y: Microvessel density as a prognostic factor in women with breast cancer: a systematic review of the literature and meta-analysis. Cancer Res 2004, 64:2941-2955.

7. Lopes N, Sousa B, Vieira D, Milanezi F, Schmitt F: Vessel density assessed by endoglin expression in breast carcinomas with different expression profiles. Histopathology 2009, 55:594-599.

8. Marinho A, Soares R, Ferro J, Lacerda M, Schmitt FC: Angiogenesis in breast cancer is related to age but not to other prognostic parameters Pathol Res Pract 1997, 193:267-273.

9. Stefansson IM, Salvesen HB, Akslen LA: Vascular proliferation is important for clinical progress of endometrial cancer. Cancer Res 2006, 66:3303-3309.

10. Naik RP, Jin D, Chuang E, Gold EG, Tousimis EA, Moore AL, Christos PJ, de Dalmas T, Donovan D, Rafii S, Vahdat LT: Circulating endothelial progenitor cells correlate to stage in patients with invasive breast cancer. Breast Cancer Res Treat 2008, 107:133-138.

11. Sørlie T, Perou C, Tibshirani R, Aas T, Geisler S, Johnsen H, Hastie T, Eisen M, van de Rijn M, Jeffrey S, Thorsen T, Quist H, Matese J, Brown P, Botstein D, Lønning PE, Børresen-Dale A: Gene expression patterns of breast carcinomas distinguish tumor subclasses with clinical implications. Proc Natl Acad Sci USA 2001, 98:10869-10874.

12. Chin K, DeVries S, Fridlyand J, Spellman PT, Roydasgupta R, Kuo W-L, Lapuk A, Neve RM, Qian Z, Ryder T, Chen F, Feiler H, Tokuyasu T, Kingsley C, Dairkee S, Meng Z, Chew K, Pinkel D, Jain A, Ljung BM, Esserman L, Albertson DG, Waldman FM, Gray JW: Genomic and transcriptional aberrations linked to breast cancer pathophysiologies. Cancer Cell 2006, 10:529-541.

13. Finak $G$, Bertos $N$, Pepin $F$, Sadekova S, Souleimanova M, Zhao $H$, Chen $H$, Omeroglu G, Meterissian S, Omeroglu A, Hallett M, Park M: Stromal gene expression predicts clinical outcome in breast cancer. Nat Med 2008, 14:518-527.

14. Chang HY, Nuyten DS, Sneddon JB, Hastie T, Tibshirani R, Sorlie T, Dai H, He YD, van't Veer LJ, Bartelink $H$, van de Rijn M, Brown PO, van de Vijver MJ: Robustness, scalability, and integration of a wound-response gene expression signature in predicting breast cancer survival. Proc Natl Acad Sci USA 2005, 102:3738-3743.

15. Chang HY, Sneddon JB, Alizadeh AA, Sood R, West RB, Montgomery K, Chi JT, van de Rijn M, Botstein D, Brown PO: Gene expression signature of fibroblast serum response predicts human cancer progression: similarities between tumors and wounds. PLOS Biol 2004, 2:E7.

16. Teschendorff AE, Miremadi A, Pinder SE, Ellis IO, Caldas C: An immune response gene expression module identifies a good prognosis subtype in estrogen receptor negative breast cancer. Genome Biol 2007, 8:R157.

17. Bhati R, Patterson C, Livasy CA, Fan C, Ketelsen D, Hu Z, Reynolds E, Tanner C, Moore DT, Gabrielli F, Perou CM, Klauber-DeMore N: Molecular characterization of human breast tumor vascular cells. Am J Pathol 2008, 172:1381-1390.

18. Parker BS, Argani P, Cook BP, Liangfeng H, Chartrand SD, Zhang M, Saha S, Bardelli A, Jiang Y, Martin TBS, Nacht M, Teicher BA, Klinger KW, Sukumar S, Madden SL: Alterations in vascular gene expression in invasive breast carcinoma. Cancer Res 2004, 64:7857-7866.

19. Croix BS, Rago C, Velculescu V, Traverso G, Romans KE, Montgomery E, Lal A, Riggins GJ, Lengauer C, Vogelstein B, Kinzler KW: Genes expressed in human tumor endothelium. Science 2000, 289:1197-1202.

20. van Beijnum JR, Dings RP, van der Linden E, Zwaans BMM, Ramaekers FCS, Mayo KH, Griffioen AW: Gene expression of tumor angiogenesis dissected: specific targeting of colon cancer angiogenic vasculature. Blood 2006, 108:2339-2348.
21. Buckanovich RJ, Sasaroli D, O'Brien-Jenkins A, Botbyl J, Hammond R, Katsaros D, Sandaltzopoulos R, Liotta LA, Gimotty PA, Coukos G: Tumor vascular proteins as biomarkers in ovarian cancer. J Clin Oncol 2007, 25:852-861.

22. Pen A, Moreno MJ, Martin J, Stanimirovic DB: Molecular markers of extracellular matrix remodeling in glioblastoma vessels: microarray study of laser-captured glioblastoma vessels. Glia 2007, 55:559-572.

23. Madden SL, Cook BP, Nacht M, Weber WD, Callahan MR, Jiang Y, Dufault MR, Zhang X, Zhang W, Walter-Yohrling J, Rouleau C, Akmaev VR, Wang CJ, Cao X, Martin TBS, Roberts BL, Teicher BA, Klinger KW, Stan R-V, Lucey B, Carson-Walter EB, Laterra J, Walter KA: Vascular gene expression in nonneoplastic and malignant brain. Am J Pathol 2004, 165:601-608.

24. Finak G, Sadekova S, Pepin F, Hallett M, Meterissian S, Halwani F, Khetani K, Souleimanova M, Zabolotny B, Omeroglu A, Park M: Gene expression signatures of morphologically normal breast tissue identify basal-like tumors. Breast Cancer Res 2006, 8:R58

25. NIH ImageJ. [http://rsb.info.nih.gov/ij]

26. Suzuki $R$, Shimodaira $H$ : Pvclust: an R package for assessing the uncertainty in hierarchical clustering. Bioinformatics 2006, 22:1540-1542.

27. Smyth GK: Limma: linear models for microarray data. In Bioinformatics and Computational Biology Solutions using R and Bioconductor. Edited by: Gentleman R, Carey V, Dudoit S, Irizarry R, Huber W. New York: Springer; 2005:397-420.

28. Benjamini $Y$, Hochberg $Y$ : Controlling the false discovery rate: a practical and powerful approach to multiple testing. J R Stat Soc Series B 1995, 57:289-300.

29. van Beijnum JR, Petersen K, Griffioen AW: Tumor endothelium is characterized by a matrix remodeling signature. Front Biosci (Schol Ed) 2009, 1:216-225.

30. Jain RK: Molecular regulation of vessel maturation. Nat Med 2003, 9:685-693

31. Baluk P, Hashizume $H$, McDonald DM: Cellular abnormalities of blood vessels as targets in cancer. Curr Opin Genet Dev 2005, 15:102-111.

32. Houle F, Huot J: Dysregulation of the endothelial cellular response to oxidative stress in cancer. Mol Carcinog 2006, 45:362-367.

33. Chlench S, Disassa NM, Hohberg M, Hoffmann C, Pohlkamp T, Beyer G, Bongrazio M, Silva-Azevedo LD, Baum O, Pries AR, Zakrzewicz A: Regulation of Foxo-1 and the angiopoietin-2/Tie2 system by shear stress. FEBS Lett 2007, 581:673-680

34. Lalor PF, Edwards S, McNab G, Salmi M, Jalkanen S, Adams DH: Vascular adhesion protein-1 mediates adhesion and transmigration of lymphocytes on human hepatic endothelial cells. J Immunol 2002, 169:983-992.

35. Fukumura D, Jain RK: Tumor microenvironment abnormalities: causes, consequences, and strategies to normalize. J Cell Biochem 2007, 101:937-949.

36. Premzl A, Turk V, Kos J: Intracellular proteolytic activity of cathepsin B is associated with capillary-like tube formation by endothelial cells in vitro. J Cell Biochem 2006, 97:1230-1240.

37. Nyberg $P$, Xie L, Kalluri R: Endogenous inhibitors of angiogenesis. Cancer Res 2005, 65:3967-3979.

38. Domenga V, Fardoux P, Lacombe P, Monet M, Maciazek J, Krebs LT, Klonjkowski B, Berrou E, Mericskay M, Li Z, Tournier-Lasserve E, Gridley T, Joutel A: Notch3 is required for arterial identity and maturation of vascular smooth muscle cells. Genes Dev 2004, 18:2730-2735.

39. Rehman $\mathrm{AO}$, Wang $\mathrm{C}-\mathrm{Y}$ : Notch signaling in the regulation of tumor angiogenesis. Trends Cell Biol 2006, 16:293-300.

40. Mitchell TS, Bradley J, Robinson GS, Shima DT, Ng Y-S: RGS5 expression is a quantitative measure of pericyte coverage of blood vessels. Angiogenesis 2008, 11:141-151.

41. Fujita M, Khazenzon NM, Bose S, Sekiguchi K, Sasaki T, Carter WG, Ljubimov AV, Black KL, Ljubimova JY: Overexpression of beta1-chain-containing laminins in capillary basement membranes of human breast cancer and its metastases. Breast Cancer Res 2005, 7:R411-R421.

42. Williams KJ, Telfer BA, Airley RE, Peters HP, Sheridan MR, van der Kogel AJ, Harris AL, Stratford IJ: A protective role for HIF-1 in response to redox manipulation and glucose deprivation: implications for tumorigenesis. Oncogene 2002, 21:282-290.

43. Song $N$, Huang $Y$, Shi $H$, Yuan S, Ding $Y$, Song $X$, Fu Y, Luo $Y$ : Overexpression of platelet-derived growth factor-BB increases tumor pericyte content via stromal-derived factor-1alpha/CXCR4 axis. Cancer Res 2009, 69:6057-6064. 
44. Eklund L, Olsen BR: Tie receptors and their angiopoietin ligands are context-dependent regulators of vascular remodeling. Exp Cell Res 2006, 312:630-641.

45. Chien S: Mechanotransduction and endothelial cell homeostasis: the wisdom of the cell. Am J Physiol Heart Circ Physiol 2007, 292:H1209-H1224

46. Ishimura N, Bronk SF, Gores GJ: Inducible nitric oxide synthase upregulates Notch-1 in mouse cholangiocytes: implications for carcinogenesis. Gastroenterology 2005, 128:1354-1368.

47. van Beijnum JR, Petersen K, Griffioen AW: Tumor endothelium is characterized by a matrix remodeling signature. Front Biosci (Schol Ed) 2009, 1:216-225.

48. Kim S, Takahashi H, Lin W-W, Descargues P, Grivennikov S, Kim Y, Luo J-L, Karin M: Carcinoma-produced factors activate myeloid cells through TLR2 to stimulate metastasis. Nature 2009, 457:102-106.

49. Laramée M, Chabot C, Cloutier M, Stenne R, Holgado-Madruga M, Wong AJ, Royal I: The scaffolding adapter Gab1 mediates vascular endothelial growth factor signaling and is required for endothelial cell migration and capillary formation. J Biol Chem 2007, 282:7758-7769.

50. Kakolyris S, Fox SB, Koukourakis M, Giatromanolaki A, Brown N, Leek RD, Taylor M, Leigh IM, Gatter KC, Harris AL: Relationship of vascular maturation in breast cancer blood vessels to vascular density and metastasis, assessed by expression of a novel basement membrane component, LH39. Br J Cancer 2000, 82:844-851.

51. Sparano JA, Paik S: Development of the 21-gene assay and its application in clinical practice and clinical trials. J Clin Oncol 2008, 26:721-728.

52. Cardoso F, Veer LV, Rutgers E, Loi S, Mook S, Piccart-Gebhart MJ: Clinical application of the 70-gene profile: the MINDACT trial. J Clin Oncol 2008, 26:729-735.

53. Igreja C, Courinha M, Cachaço AS, Pereira T, Cabeçadas J, da Silva MG Dias S: Characterization and clinical relevance of circulating and biopsyderived endothelial progenitor cells in lymphoma patients. Haematologica 2007, 92:469-477.

54. Ebos JM, Lee CR, Cruz-Munoz W, Bjarnason GA, Christensen JG, Kerbel RS: Accelerated metastasis after short-term treatment with a potent inhibitor of tumor angiogenesis. Cancer Cell 2009, 15:232-239.

55. Sennino B, Kuhnert F, Tabruyn SP, Mancuso MR, Hu-Lowe DD, Kuo CJ, McDonald DM: Cellular source and amount of vascular endothelial growth factor and platelet-derived growth factor in tumors determine response to angiogenesis inhibitors. Cancer Res 2009, 69:4527-4536.

56. Joyce JA: Therapeutic targeting of the tumor microenvironment. Cancer Cell 2005, 7:513-520

57. Gordon MS, Mendelson DS, Kato G: Tumor angiogenesis and novel antiangiogenic strategies. Int J Cancer 2010, 126:1777-1787.

58. You WK, McDonald DM: The hepatocyte growth factor/c-Met signaling pathway as a therapeutic target to inhibit angiogenesis. BMB Rep 2008, 41:833-839.

59. Santulli RJ, Kinney WA, Ghosh S, Decorte BL, Liu L, Tuman RW, Zhou Z, Huebert N, Bursell SE, Clermont AC, Grant MB, Shaw LC, Mousa SA, Galemmo RA Jr, Johnson DL, Maryanoff BE, Damiano BP: Studies with an orally bioavailable alpha $\mathrm{V}$ integrin antagonist in animal models of ocular vasculopathy: retinal neovascularization in mice and retinal vascular permeability in diabetic rats. J Pharmacol Exp Ther 2008, 324:894-901

60. Helfrich I, Scheffrahn I, Bartling S, Weis J, von Felbert V, Middleton M, Kato M, Ergun S, Schadendorf D: Resistance to antiangiogenic therapy is directed by vascular phenotype, vessel stabilization, and maturation in malignant melanoma. J Exp Med 2010, 207:491-503.

61. Mazzone M, Dettori D, Leite de Oliveira R, Loges $S$, Schmidt T, Jonckx B, Tian YM, Lanahan AA, Pollard P, Ruiz de Almodovar C, De Smet F, Vinckier S, Aragones J, Debackere K, Luttun A, Wyns S, Jordan B, Pisacane A, Gallez B, Lampugnani MG, Dejana E, Simons M, Ratcliffe P, Maxwell P, Carmeliet P: Heterozygous deficiency of PHD2 restores tumor oxygenation and inhibits metastasis via endothelial normalization. Cell 2009, 136:839-851.

62. Jain RK, Duda DG, Willett CG, Sahani DV, Zhu AX, Loeffler JS, Batchelor T, Sorensen AG: Biomarkers of response and resistance to antiangiogenic therapy. Nat Rev Clin Oncol 2009, 6:327-338.

doi: $10.1186 / \mathrm{bcr} 3246$

Cite this article as: Pepin et al: Gene-expression profiling of microdissected breast cancer microvasculature identifies distinct tumor vascular subtypes. Breast Cancer Research 2012 14:R120.

\section{Submit your next manuscript to BioMed Central and take full advantage of:}

- Convenient online submission

- Thorough peer review

- No space constraints or color figure charges

- Immediate publication on acceptance

- Inclusion in PubMed, CAS, Scopus and Google Scholar

- Research which is freely available for redistribution

Submit your manuscript at www.biomedcentral.com/submit
C Biomed Central 